\title{
BMJ Open E-cigarette use and combustible tobacco cigarette smoking uptake among non- smokers, including relapse in former smokers: umbrella review, systematic review and meta-analysis
}

\author{
Olivia Nina Baenziger (D) , ${ }^{1}$ Laura Ford, ${ }^{2}$ Amelia Yazidjoglou (D) , ${ }^{2}$ Grace Joshy (D) , ${ }^{2}$ \\ Emily Banks (D) ${ }^{2}$
}

To cite: Baenziger $\mathrm{ON}$, Ford $\mathrm{L}$, Yazidjoglou A, et al. E-cigarette use and combustible tobacco cigarette smoking uptake among non-smokers, including relapse in former smokers: umbrella review, systematic review and meta-analysis. BMJ Open 2021;11:e045603. doi:10.1136/ bmjopen-2020-045603

- Prepublication history and additional materials for this paper is available online. To view these files, please visit the journal online (http://dx.doi org/10.1136/bmjopen-2020045603).

Received 07 October 2020 Revised 01 March 2021 Accepted 14 March 2021

Check for updates

(c) Author(s) (or their employer(s)) 2021. Re-use permitted under CC BY-NC. No commercial re-use. See rights and permissions. Published by BMJ.

${ }^{1}$ The University of Melbourne School of Population and Global Health, Melbourne, Victoria, Australia

${ }^{2}$ The National Centre for Epidemiology and Population Health, Australian National University, Canberra, Australian Capital Territory, Australia

Correspondence to Professor Emily Banks; emily.banks@anu.edu.au

\section{ABSTRACT}

Objective To review and summarise the current evidence on the uptake of combustible cigarette smoking following e-cigarette use in non-smokers-including never-smokers, people not currently smoking and past smokers-through an umbrella review, systematic review and meta-analysis.

Design Umbrella review, systematic review and metaanalysis.

Data sources PubMed, Scopus, Web of Science, PsychINFO (Ovid), Medline (Ovid) and Wiley Cochrane Library up to April 2020.

Results Of 6225 results, 25 studies of non-smokersnever, not current and former smokers - with a baseline measure of e-cigarette use and an outcome measure of combustible smoking uptake were included. All 25 studies found increased risk of smoking uptake with e-cigarette exposure, although magnitude varied substantially. Using a random-effects model, comparing e-cigarette users versus non-e-cigarette users, among never-smokers at baseline the OR for smoking initiation was $3.19(95 \% \mathrm{Cl} 2.44$ to $\left.4.16, I^{2} 85.7 \%\right)$ and among non-smokers at baseline the OR for current smoking was $3.14(95 \% \mathrm{Cl} 1.93$ to 5.11 , $I^{2}$ 91.0\%). Among former smokers, smoking relapse was higher in e-cigarette users versus non-users $(\mathrm{OR}=2.40$, $95 \% \mathrm{Cl} 1.50$ to $\left.3.83, \mathrm{I}^{2} 12.3 \%\right)$.

Conclusions Across multiple settings, non-smokers who use e-cigarettes are consistently more likely than those avoiding e-cigarettes to initiate combustible cigarette smoking and become current smokers. The magnitude of this risk varied, with an average of around three times the odds. Former smokers using e-cigarettes have over twice the odds of relapse as non-e-cigarettes users. This study is the first to our knowledge to review and pool data on the latter topic.

PROSPERO registration number CRD42020168596.

\section{INTRODUCTION}

Globally, combustible tobacco smoking results in over 8 million deaths each year. ${ }^{1}$ Due to vigorous public health interventions, smoking prevalence in Australia has declined

\section{Strengths and limitations of this study}

- Comprehensive and systematic literature search with pooled evidence from 25 published studies reviewed according to a prespecified protocol.

- Inclusion of studies investigating all ages and types of non-smokers (never, not current and former).

- Independent corroboration of results from previous studies, reviews and meta-analyses, while adding evidence on smoking uptake with e-cigarette exposure among former smokers.

- The evidence is largely reliant on self-reported product use and the studies reviewed were observational in nature as it is not ethical or appropriate to randomise non-smokers to e-cigarette exposure.

- While all studies reported significantly higher uptake of tobacco smoking among non-smokers exposed to e-cigarettes, compared with those not exposed, there was significant variation in the magnitude of the observed increase in risk; the results of the meta-analyses should therefore be considered to be an average of the published studies.

substantially over the last 50 years. $^{2}$ Nevertheless, $9.3 \%$ of the total disease burden (in disability-adjusted life years) was attributable to combustible tobacco use in $2015 .^{3}$

E-cigarettes are a diverse group of batteryoperated or rechargeable devices that heat a liquid ('e-liquid' or 'e-juice') to produce a vapour that users inhale. Although the composition of e-liquid varies, it typically contains a range of chemicals including propylene glycol and flavouring agents and are commonly used to deliver nicotine. ${ }^{4}$ The labelling of electronic nicotine delivery systems (ENDS) and electronic non-nicotine delivery systems (ENNDS) is not always accurate, with reports of nicotine found in products labelled ENNDS. ${ }^{45}$ 
Studies indicate that in many countries, e-cigarette use among never-smoking youth is increasing. ${ }^{6-11}$ In Australia, the proportion of non-smokers aged 14 years or older who had ever used e-cigarettes increased from $4.9 \%$ in 2016 to $6.9 \%$ in $2019 .{ }^{12}$ The increase was particularly notable in young adults, with $20 \%$ of $18-24$ years old non-smokers reporting e-cigarette use. ${ }^{12}$ E-cigarette use among youth is predominantly driven by curiosity and experimentation rather than smoking cessation. ${ }^{13-15}$ Evidence also suggests that most people who report ever e-cigarette do not graduate to regular e-cigarette use. ${ }^{1516}$ Although the identification of risk factors for initiation of e-cigarette use is complex, it appears as though many are similar to those for smoking initiation. ${ }^{1718}$

There are concerns that the use of e-cigarettes in neversmokers may increase the probability that they will try combustible tobacco cigarettes and go on to become regular smokers, particularly among youth and young adults. ${ }^{19}{ }^{20}$ Furthermore, use of e-cigarettes could conceivably lead to combustible tobacco smoking relapse in former smokers. If e-cigarette use leads to more people smoking combustible cigarettes, compared with the number of people who have smoked in the absence of e-cigarettes, this would be a source of considerable public health harm. ${ }^{21}$ Thus, our primary research question is: among never smokers, current non-smokers and former smokers, how does e-cigarette use affect the subsequent risk of initiating use, current use and relapse to combustible tobacco cigarettes? This review aims to systematically update global contemporary population-level evidence on the relationship of e-cigarette use to smoking uptake.

\section{METHODS}

This summary of the global evidence comprises an umbrella review of systematic reviews and a top-up systematic review of primary research not included in the systematic reviews of the umbrella review. The protocol was published online through PROSPERO.

\section{Search strategy}

The Population, Intervention, Comparison, Outcome (PICO) format was used to structure the search (online supplemental table 1). Studies investigating the association between ENDS or ENNDS use among non-tobacco smokers and uptake of combustible cigarette smoking were included. E-cigarette use, cigarette smoking and uptake related search terms and keywords were used (online supplemental table 2). For both the umbrella review and the top-up systematic review, six databases (PubMed, Scopus, Web of Science, PsycINFO (Ovid), MEDLINE (Ovid) and Cochrane) were searched on 1 April 2020 (online supplemental table 3).

\section{Inclusion and exclusion criteria}

Systematic reviews and meta-analyses of prospective cohort studies or randomised or non-randomised controlled trials (RCTs) examining the exposure (e-cigarette use) and outcome (smoking uptake in current non-smokers) of interest were included in the umbrella review. For the top-up systematic review, individual prospective cohort studies or randomised or non-RCTs identified in the search and not included in the umbrella review studies, were included. Cross-sectional studies were excluded due to difficulties in establishing the temporal relationship between e-cigarette exposure and smoking uptake. Studies with a follow-up of less than 6 months or with abstracts not published in English were excluded. The full inclusion and exclusion criteria can be found in online supplemental table 1.

\section{Data screening and extraction}

EndNote and Covidence software were used for review management. Two authors of this review (ONB and LF) undertook initial screening, study selection, risk of bias assessment and data extraction. Titles and abstracts identified in the searches were screened using a checklist, followed by full-text screening. A forward and backward reference search using Scopus was performed from the final included articles. After removing duplicates, titles, abstracts and then full texts were screened for any studies fulfilling the inclusion and exclusion criteria. Data were independently extracted from the included systematic reviews and cohort studies using a prespecified data extraction template. As it is important to consider whether authors of the studies under review hold any conflicts of interest that could potentially bias their findings, or whether the research was funded by an organisation with a financial interest in the outcomes, information on the source of research sponsorship or external involvement was also extracted. Studies were considered separately if they received funding from the tobacco or nicotine industry.

\section{Risk of bias assessment}

Risk of bias for each study included was independently assessed using the AMSTAR $2^{22}$ for the systematic reviews and meta-analyses included in the umbrella reviews, and the Newcastle-Ottawa Scale (NOS) ${ }^{23}$ for the studies in the top-up systematic review. For meta-analyses with at least 10 studies, risk of bias across studies was assessed and interpreted using the symmetry of funnel plots and superimposed $95 \%$ confidence limits. $^{24}$

\section{Summary measures and synthesis of results}

Findings from the umbrella review and the top-up systematic review were synthesised separately in narrative summaries. Individual prospective primary research studies identified from both the umbrella review and top-up systematic review were then considered in an integrated systematic review. Where appropriate, ORs from the studies in the integrated systematic review were combined using a random-effects model. Heterogeneity of study effect estimates were assessed by an $\mathrm{I}^{2}$ statistic. All analyses were conducted using Stata V.16.1. 


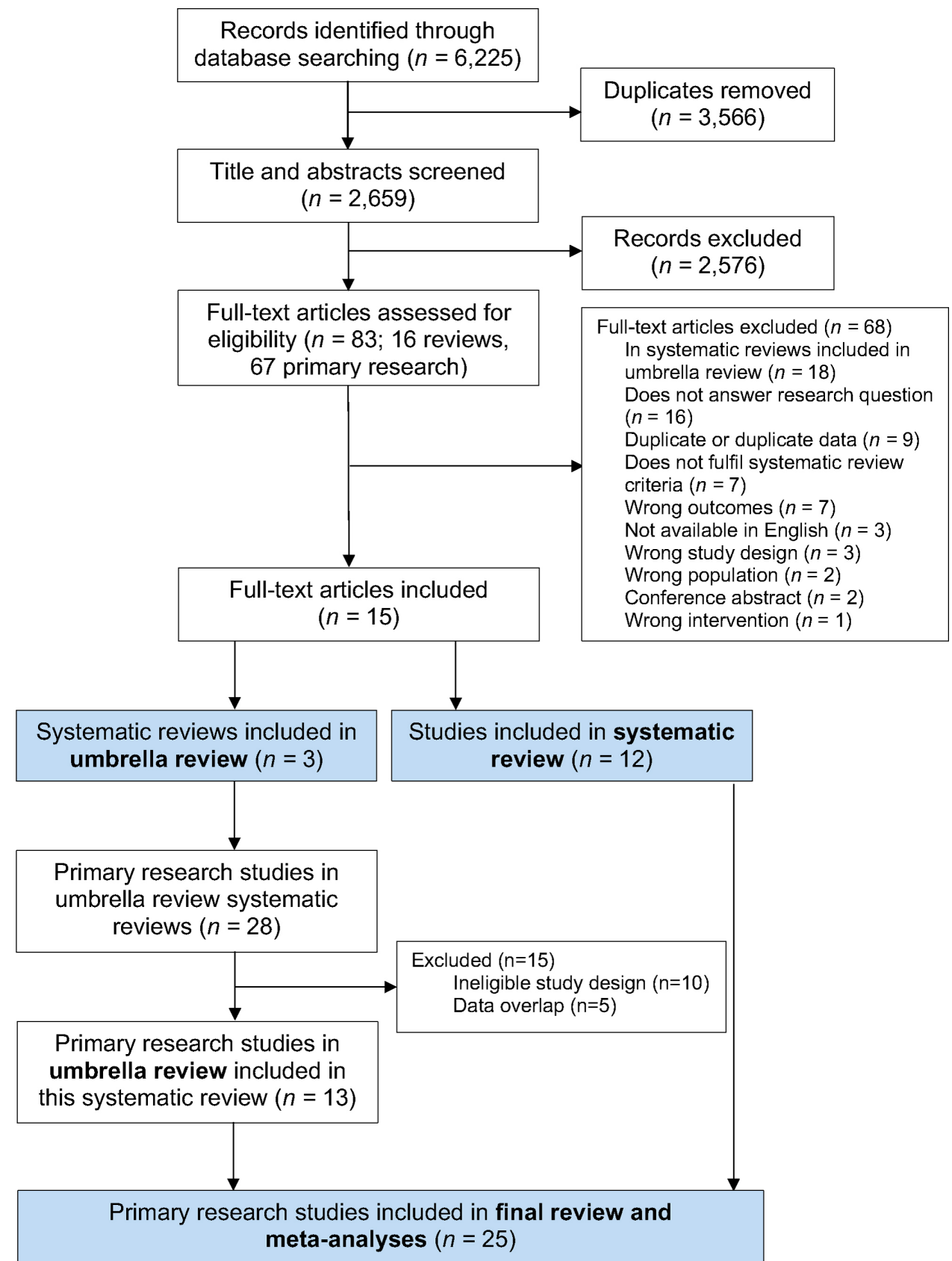

Figure 1 Flow chart for selection of studies for inclusion in umbrella review and top-up systematic review.

Patient and public involvement

No patient involved.

\section{RESULTS}

\section{Study selection}

Study selection for this umbrella review and top-up systematic review are shown in the Preferred Reporting Items for Systematic Reviews and Meta-Analyses flowchart in figure 1. A total of 6225 studies were identified for title and abstract screening; 2659 remained after exclusion of duplicates. After title and abstract screening, 83 articles were identified for full-text screening. Fifteen papers were identified for inclusion; three were systematic reviews that were included in the umbrella review and 12 were primary research studies included in the top-up systematic review. Ten of the latter studies were prospective observational studies and two were secondary analyses of RCTs.

From the three systematic review papers included in the umbrella review, 28 primary research studies were identified after removing duplicates. For our meta-analyses, we excluded 15 studies due to ineligible study design $(n=10)$ or data overlap $(n=5)$. No studies were excluded based on their quality assessment scores. The meta-analyses were thus based on 13 primary research studies identified from the prior systematic reviews, and 12 studies from our top-up systematic review, that is, a total of 25 primary research studies on e-cigarette use and smoking uptake (figure 1). 
Table 1 ORs and adjusted ORs of the association between e-cigarette use and combustible cigarette smoking from systematic reviews and meta-analyses included in the umbrella review

\begin{tabular}{|c|c|c|c|}
\hline Authors/year & $\begin{array}{l}\text { Studies included } \\
\text { ( } \mathrm{n}=\text { total population) }\end{array}$ & OR $(95 \% \mathrm{Cl})$ & $\begin{array}{l}\text { Adjusted OR }(95 \% \mathrm{Cl}) \text { and } \\
\text { heterogeneity }\left(\mathrm{I}^{2}\right)\end{array}$ \\
\hline Khouja et $a l^{43}$ & $17(n=105448)$ & 4.59 (3.60 to 5.85$)$ & $2.92(2.30$ to 3.71$) I^{2}: 84.5 \%$ \\
\hline Soneji et $a l^{21}$ & $9(n=17389)$ & $\begin{array}{l}\text { Initiation: } 3.83 \text { (3.74 to } 3.91 \text { ) } \\
\text { Past } 30 \text {-day: } 5.68 \text { (3.49 to } 9.24 \text { ) }\end{array}$ & $\begin{array}{l}\text { Initiation: } 3.50 \text { (2.38 to } 5.16 \text { ) } I^{2}: 56 \% \\
\text { Past } 30 \text {-day: } 4.28 \text { (2.52 to } 7.27) I^{2}: 0 \%\end{array}$ \\
\hline
\end{tabular}

No potential competing interests were identified in the included studies themselves, or by the authors, based on the disclosure statements from the publications. Although one ${ }^{25}$ primary research study identified during screening in the top-up systematic review was found to have potential competing interests, as it was funded by the tobacco industry, it was previously excluded due to a large overlap with data presented in a more recent paper by Berry et $a l^{26}$

There is considerable uncertainty regarding the chemical constituents of the e-liquids delivered by the e-cigarettes in the studies included in the review. Where evidence on nicotine content was available, it indicated that a substantial majority of e-cigarettes in those studies delivered nicotine. ${ }^{27-30}$ Many publications noted considerable uncertainty regarding nicotine content, including apparent mislabelling, and the need for greater clarity and reliability on this point.

\section{Umbrella review: quality assessment}

All three systematic reviews from the selected articles rated moderate in the AMSTAR $2^{22}$ assessment. Information was lacking regarding study exclusion criteria, stated sources of funding and detail on data extraction (online supplemental table 4).

\section{Umbrella review}

Table 1 summarises the results of the three systematic reviews included in the umbrella review. All three systematic reviews excluded studies with participants over 30 years of age. Sample sizes for the individual studies varied considerably, ranging from 298 to 17318 . Of the 13 included longitudinal primary research studies (detailed in online supplemental table 5), $9^{2031-38}$ were based in the USA, $2^{39} 40$ in the UK and 1 each in Mexico, ${ }^{41}$ and the Netherlands. ${ }^{42}$ Each of the three systematic reviews conducted meta-analyses and found the odds of smoking initiation were increased for youth and young adult e-cigarette users compared with non-e-cigarette users; these results are summarised in table 1 .

The Khouja et al systematic review and meta-analysis included 17 studies published up to November $2018 .^{43}$ The study found that the risk of later smoking in people aged $<30$ years who had ever used or currently use e-cigarettes was strong; an almost threefold the odds compared with never users after adjustment for covariates (see table 1). However, there were high levels of heterogeneity in the summary estimates (adjusted OR $\mathrm{I}^{2}=84.5 \%$ ), which remained high in adjusted analysis subgrouping by age, ever smoking, risk of bias and location of study. Heterogeneity was reduced when the adjusted ORs were grouped into those examining the relationship between ever e-cigarette use and current smoking (adjusted OR 2.21; $95 \%$ CI 1.72 to $2.84, \mathrm{I}^{2}=5 \%$ ) and those assessing the relationship of current e-cigarette use to ever smoking (adjusted OR 2.33; $95 \%$ CI 1.84 to $2.96, \mathrm{I}^{2}=5 \%$ ).

Aladeokin and Haighton aimed to systematically review the evidence on e-cigarette use and initiation of cigarette smoking in adolescents (aged 10-19 years old) in the UK and included eight studies. ${ }^{44}$ Their meta-analysis showed e-cigarette users were much more likely than non-users to go on to smoke combustible cigarettes, even after adjusting for covariates (see table 1); the substantial heterogeneity in the summary estimate should be noted.

The Soneji et al systematic review and meta-analysis included nine longitudinal studies of US participants $\leq 30$ years of age. ${ }^{21}$ Seven of the included studies assessed the association of baseline ever e-cigarette use with subsequent ever combustible cigarette use at follow-up among baseline never smokers. Soneji et al also identified two studies that assessed baseline past 30-day e-cigarette use with subsequent past 30-day combustible cigarette use among those reporting no past 30-day use of cigarettes at baseline. The meta-analysis showed a markedly higher odds of combustible cigarette use in those who had used e-cigarettes (table 1).

\section{Top-up systematic review: quality assessment}

The quality of the included studies was evaluated using the NOS. ${ }^{23}$ Of the 12 studies, the NOS totals (out of 10 stars) ranged from 5 to 8 (online supplemental table 6). Only one ${ }^{45}$ study rated 5, five ${ }^{28-304647}$ rated 6, two 948 rated 7 and four ${ }^{26-51}$ rated 8 . No studies received a star for assessment of outcome. The main areas impacting the NOS scores were ascertainment of exposure and adequacy of follow-up of cohorts (studies with less than $30 \%$ loss to follow-up were considered adequate).

Top-up systematic review and integration with primary research studies from the umbrella review

A total of 12 studies published in 2018, 2019 and 2020 were newly identified for the top-up systematic review (table 2; online supplemental table 7). Among the 12 included, 6 were from the USA, 2 from the UK and 1 
Table 2 ORs and adjusted ORs of the association between e-cigarette use and subsequent combustible cigarette use for: (1) never-smokers at baseline, (2) non-smokers* (never or no current use) at baseline and (3) former smokers at baseline

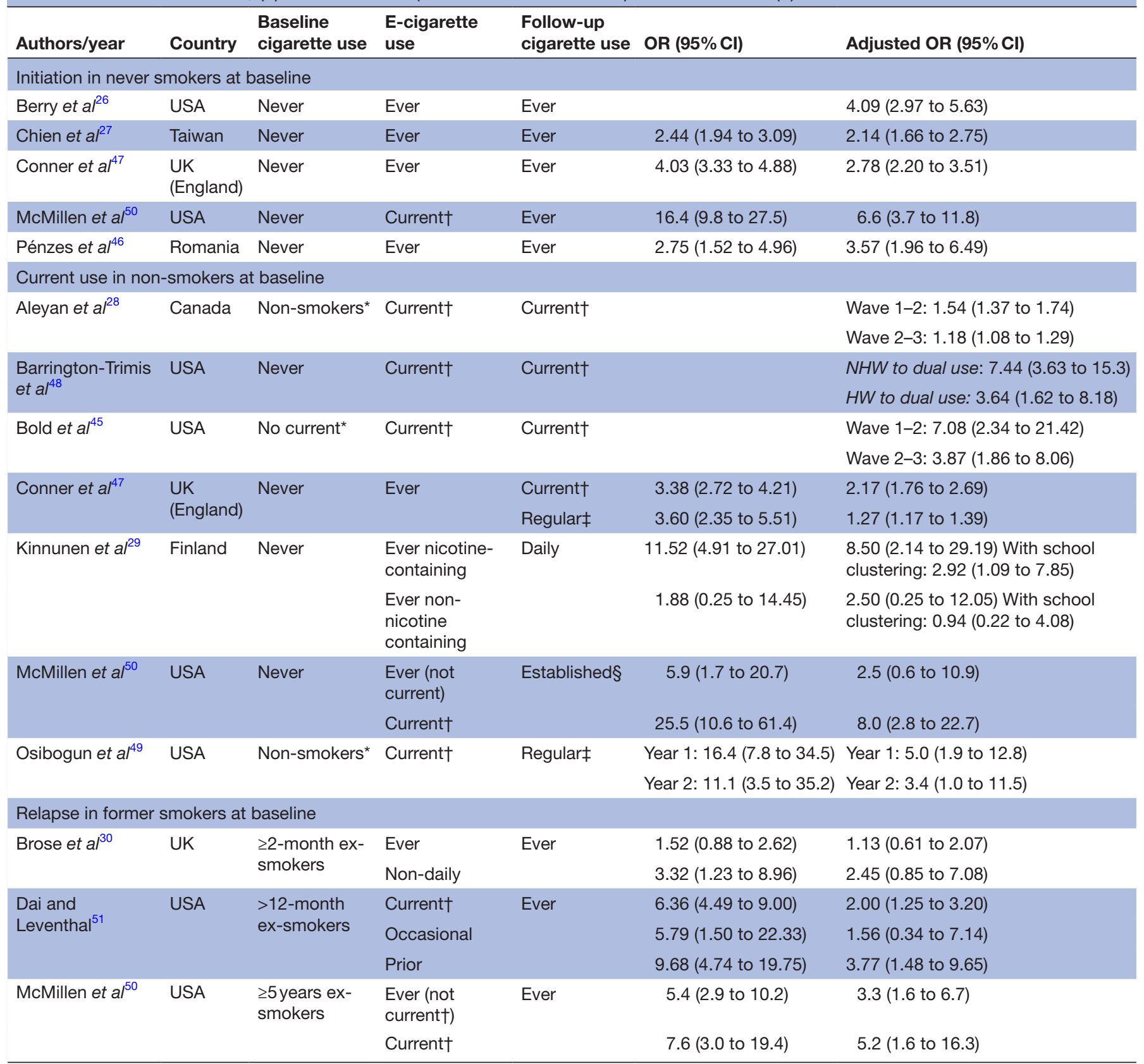

${ }^{*}$ Non-smokers defined as never or no current (past 30-day) use.

†Current defined as past 30-day use.

†Regular defined as $\geq 20$ days $/ 30$ days.

$\S$ Established defined as $\geq 100$ combustible cigarettes and currently smokes every day or some.

HW, Hispanic white; NHW, non-Hispanic white.

each from Romania, Finland, Taiwan and Canada. Study sample sizes varied considerably, ranging from 374 to 14623.

Of the six newly identified studies based on US participants, four ${ }^{2649-51}$ used Population Assessment of Tobacco and Health (PATH) data from a US nationally representative longitudinal study. Of these, two ${ }^{50}{ }^{51}$ looked at adult ( $\geq 18$ years old) former smokers, one ${ }^{49}$ looked at youth (12-17 years old) and one ${ }^{26}$ at a more restricted youth group (12-15 years old). Even though these four studies have the same data source, they were all included in this review as they had different outcome or exposure variables, different populations and included the most recent data.

Of the 12 newly identified studies, five ${ }^{2627464750}$ had outcomes assessing ever smoking among never smokers at baseline, seven ${ }^{28} 29455^{47-50}$ had outcomes assessing current smoking among non-smokers (never or not 
current smoking) at baseline and three $e^{3050}$ assessed the odds of relapse in former smokers. Results were separated based on these three categories and combined with the 13 primary research studies identified in the umbrella review. Twelve of the seventeen studies in Khouja et al were included, ${ }^{20} 31{ }^{33-42}$ three were excluded due to data overlap, ${ }^{52-54}$ one was excluded as it used retrospective data $^{55}$ and one was excluded as it was cross-sectional. ${ }^{56}$ Of the eight studies in Aladeokin and Haighton, two were included $^{3940}$; five were excluded for cross-sectional $\operatorname{design}^{57-61}$ and one for data overlap. ${ }^{54}$ From the nine studies identified in Soneji et al six were included ${ }^{31-34} 3637$ after two were excluded as they were abstracts and one excluded for data overlap. ${ }^{62}$

Cigarette smoking initiation among never smokers at baseline Five 2627464750 of the newly identified studies investigated smoking initiation among never smokers, of which Berry et $a l^{26}$ and McMillen et a $\tilde{l}^{0}$ used PATH data, focusing on youth (12-15 years old) and adults ( $\geq 18$ years old), respectively (table 2 ). Chien et al examined the association between ever e-cigarette and subsequent combustible smoking initiation in 12954 youth enrolled in schools in Taiwan between 2014 and 2016. ${ }^{27}$ Conner et al investigated the association of e-cigarette use at baseline and smoking in adolescents (13-14 years old) between waves 3 and 5 (2014-2016) of a cluster RCT in 20 schools in England. ${ }^{47}$ Pénzes et al conducted secondary data analysis from 1369 ninth grade students in the Romanian ASPIRA RCT. Details of the studies are given in online supplemental table $7 .^{46}$.

All newly identified studies found that people who used e-cigarettes were significantly more likely than non-users to initiate smoking of combustible cigarettes, with ORs varying substantially from 2.1 to $6.6\left(\mathrm{I}^{2}=81 \%\right.$; figure 2$)$.

Considering these newly identified studies along with 12 studies from the umbrella review, all found significantly increased risk of initiating smoking of combustible cigarettes in people who had used e-cigarettes, compared with those who had not (figure 2). Combining the studies from the umbrella review with the newly identified

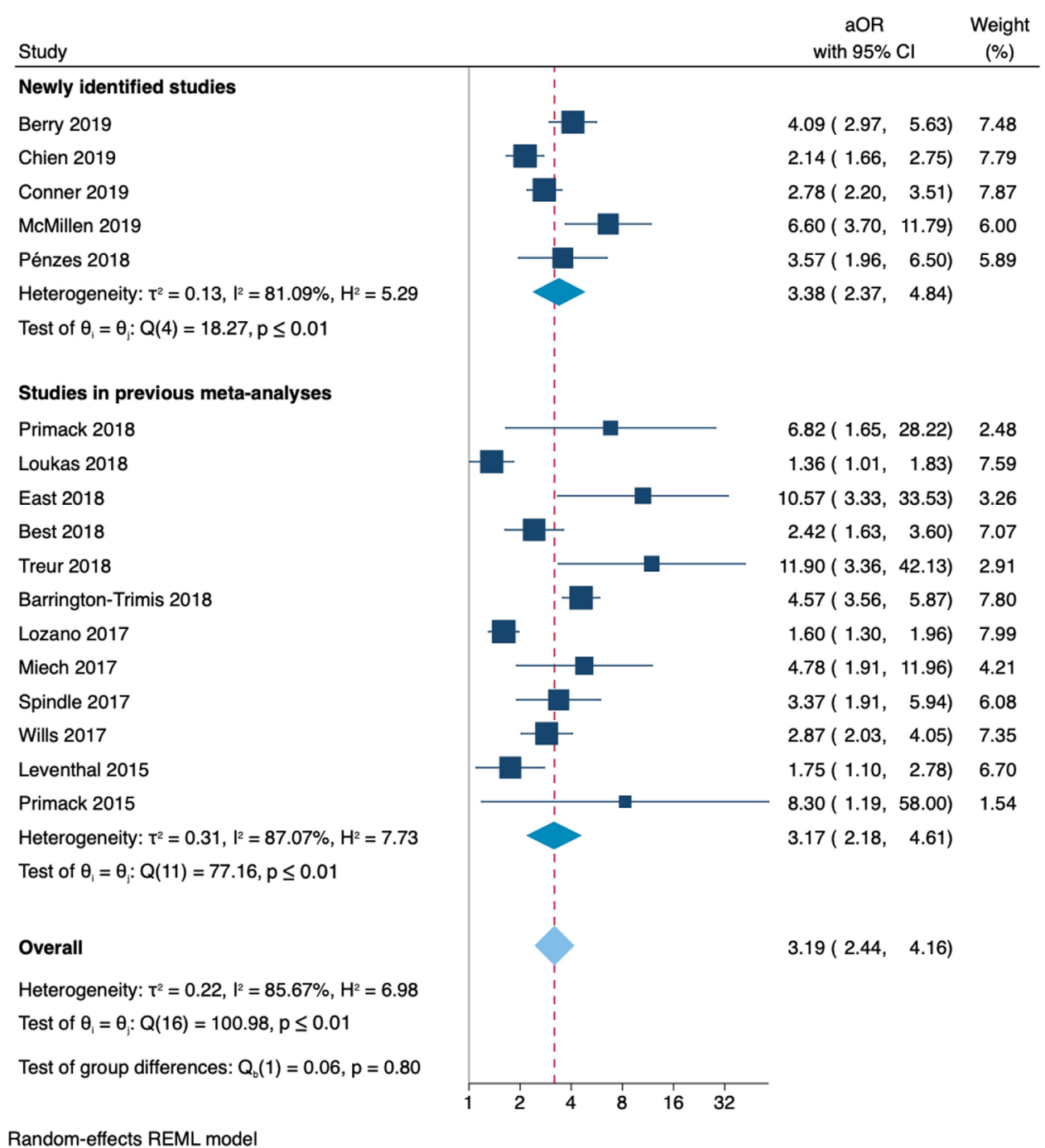

Figure 2 Forest plot and random-effects meta-analysis for the adjusted odds of smoking initiation at follow-up among never smokers and current e-cigarette users at baseline compared with never e-cigarette users at baseline. aOR, adjusted OR; REML, Restricted Maximum Likelihood 
studies, people exposed to e-cigarettes more likely to take up smoking of combustible cigarettes than people who were not exposed to e-cigarettes (pooled adjusted OR 3.19 (95\% CI 2.44 to 4.16$)$ ).

Current (past 30-day) cigarette smoking among non-smokers (never smokers or no current use at baseline) Seven $^{28} 294547-50$ of the newly identified primary research studies investigated current (past 30-day) use of combustible cigarettes following the use of e-cigarettes (table 2). Four 29474850 of these studies looked at never smokers at baseline, while three ${ }^{284549}$ looked at non-smokers (either never or no current use).

Two $^{49} 50$ of the included studies were based on PATH data. McMillen et a $\tilde{l}^{0}$ used data on adult ( $\geq 18$ years old) never smokers from waves 1 to 2 of the PATH study and Osibogun et $a t^{49}$ used data on youth (12-17 years old) non-smokers from waves 1 to 3 . A further two ${ }^{45}$ of the newly identified studies used data from the USA. Bold et al surveyed 808 high school students across three waves (2013-2015) in Connecticut. ${ }^{45}$ Barrington-Trimis et al collated data on 6258 youth from three US school-based studies between 2013 and 2015: the Children's Health Study; the Happiness and Health Study and the Yale Adolescent Survey Study. ${ }^{48}$ This study separated results based on ethnicity and found the adjusted odds of dual use at follow-up was considerably higher in non-Hispanic whites compared with Hispanic whites (see table 2), although with considerable overlap in the CIs.
The remaining three 282947 newly identified studies used data from Canada, the UK and Finland. Aleyan et al examined the association between current e-cigarette use and subsequent current smoking among 6729 Canadian school students using data from a school-based longitudinal cohort study, COMPASS. ${ }^{28}$ Conner et al investigated the association of e-cigarette use at baseline and smoking between waves 3 and 5 (2014-2016) of a cluster RCT assessing a self-regulation anti-smoking intervention from 20 schools in England. ${ }^{47}$ Kinnunen et al used MEtLoFIN a schoolbased longitudinal cohort dataset in 3474 Finnish adolescents between 2014 and 2016. ${ }^{29}$ Kinnunen et al separated the use of e-cigarettes based on their nicotine delivery and found among baseline never-smokers, ever use of nicotinedelivering e-cigarettes was associated with a nearly threefold increase in the odds of uptake of daily smoking (see table 2) and found no increase in risk associated with use of non-nicotine delivering e-cigarettes.

All of the newly identified studies, and the one relevant study from the umbrella review, ${ }^{32}$ found a significant increase in the risk of transitioning from being a nonsmoker to a current smoker in people who had used e-cigarettes compared with not using e-cigarettes, but with considerable heterogeneity in the estimates $\left(\mathrm{I}^{2}=91 \%\right.$; figure 3).

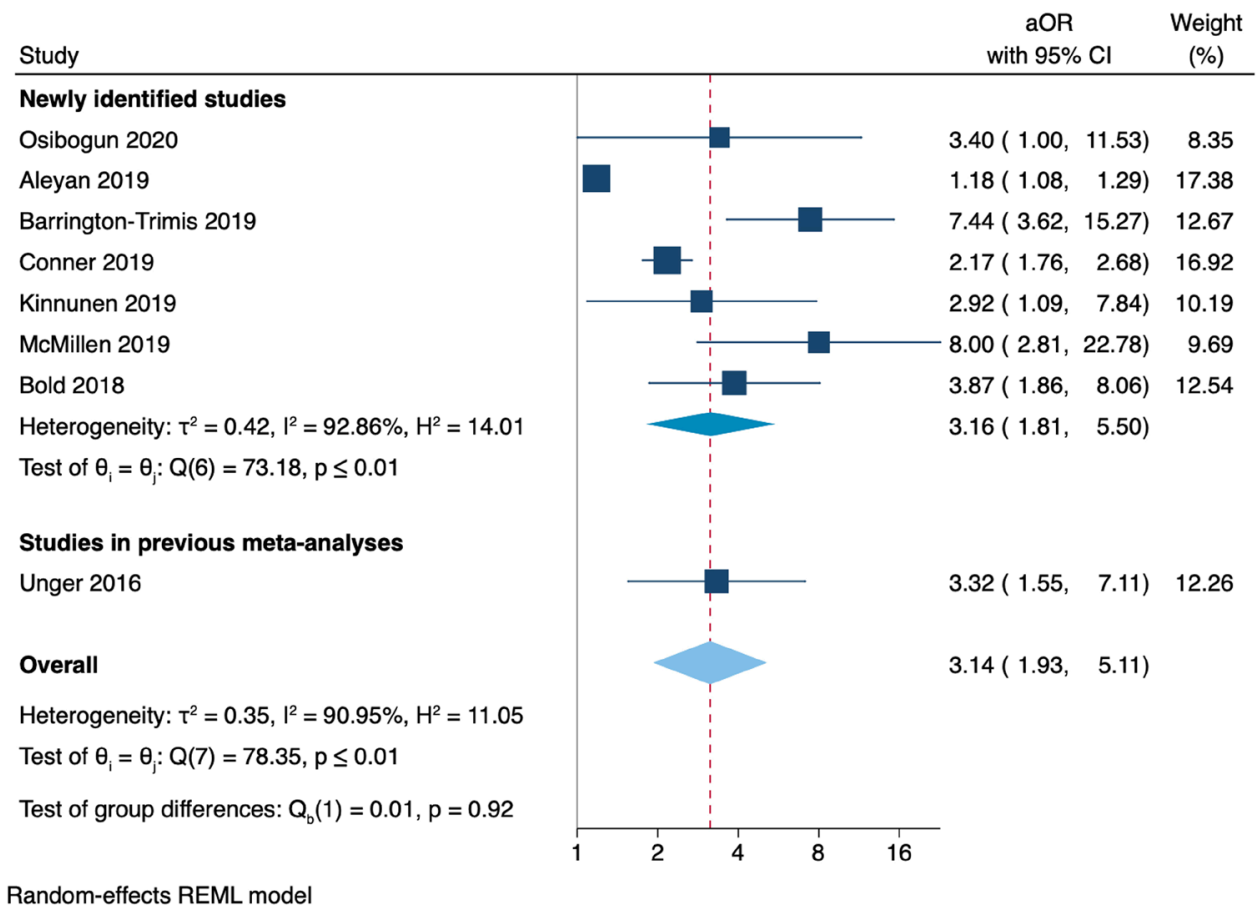

Figure 3 Forest plot and random-effects meta-analysis for the adjusted odds of current (past 30-day) smoking at follow-up among non-current smokers and current e-cigarette users at baseline compared with non-current e-cigarette users at baseline. aOR, adjusted OR; REML, Restricted Maximum Likelihood 


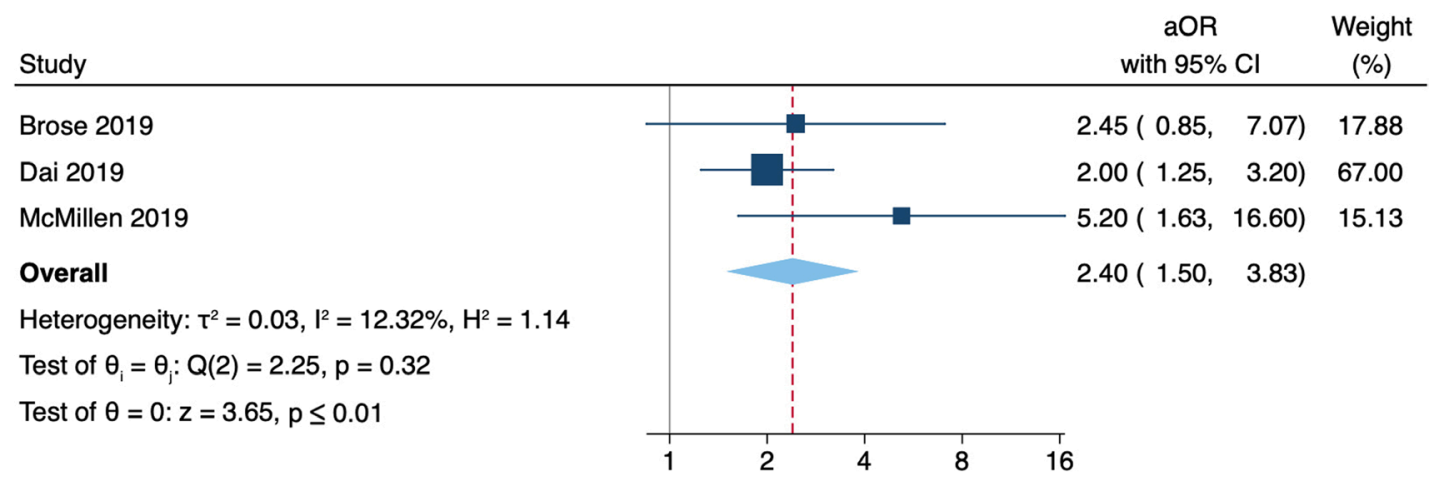

Random-effects REML model

Figure 4 Forest plot and random-effects meta-analysis for the adjusted odds of smoking relapse at follow-up among former smokers and current e-cigarette users at baseline compared with never e-cigarette users at baseline. aOR, adjusted OR; REML, Restricted Maximum Likelihood

\section{Cigarette smoking relapse among former smokers (at least 2} months since quit date)

Three ${ }^{305051}$ newly identified studies in this review investigated the odds of relapse to combustible cigarette smoking following the use of e-cigarettes in adults aged $\geq 18$ years (table 2). None of the three previously conducted systematic reviews investigated this relationship, so no additional studies from the umbrella review were included. Brose et al used data from 371 adults who quit $\geq 2$ months prior to baseline in 2016 from a national web-based survey in the UK ${ }^{30}$ The other two studies used PATH data. Dai and Leventhal looked at 3210 ex-smokers, who had not smoked for $>12$ months. ${ }^{51}$ McMillen et al looked at data relating to 8108 adults who had quit $\geq 5$ years prior to baseline; subanalyses from this study were included in the previous two sections, as the study also provided data on never smokers. ${ }^{50}$

All three included studies found the odds of ever relapse was higher among ever e-cigarette users, compared with never e-cigarette users (figure 4 ). With respect to more detailed findings, in addition to the prespecified metaanalyes, Brose et al reported lower odds of relapse among recent ex-smokers who vaped daily versus those who vaped non-daily, while Dai and Leventhal and McMillen et al showed past 30-day regular e-cigarette use had greater odds of relapse than non-current use. ${ }^{3050}$ Within the Dai and Leventhal study, regular e-cigarette use in recent smokers (quit $\leq 12$ months) was not associated with smoking relapse ${ }^{51}$ However, regular e-cigarette use in those who had ceased smoking for more than 12 months was associated with a significant increase in the odds of relapse. A metaanalysis of the three newly identified studies found former smokers who used e-cigarettes had 2.4 times greater odds of relapse when compared with those who did not use e-cigarettes, with similar magnitudes of this relationship between studies $\left(\mathrm{I}^{2}=12 \%\right)$ (figure 4$)$.

\section{Risk of bias across studies}

Funnel plots corresponding to the studies included in the meta-analyses are presented in online supplemental figure 1 . The plot for the 17 smoking initiation studies of never-smokers is somewhat asymmetrical and seven points lie outside the $95 \%$ confidence region, suggesting there may be some selection bias across included studies, publication bias or possible heterogeneity (as supported by the $\mathrm{I}^{2}$ statistic; $86 \%$ ). With less than ten studies investigating current smoking in non-smokers ${ }^{28} 29324547-49$ and relapse in former smokers, ${ }^{30} 5051$ test for funnel plot asymmetry was not used as the power of the test would be too low for it to be a reliable indicator of publication bias. ${ }^{24}$

\section{DISCUSSION}

Our umbrella and systematic review, along with an updated meta-analysis using data from primary studies, shows strong and consistent evidence that never smokers who have used e-cigarettes are more likely than those who have not used e-cigarettes to try smoking conventional cigarettes and to transition to become regular tobacco smokers. We found that, on average, non-smokers who used e-cigarettes have around threefold the odds of either initiating smoking or currently smoking combustible cigarettes compared with non-smokers who have not used e-cigarettes. The limited available evidence indicates that former smokers who report current e-cigarette use within the previous 30-days have more than twice the odds of relapse and resumption of current smoking compared with former smokers who have not used e-cigarettes.

This review builds on and has findings consistent with earlier systematic reviews and meta-analyses in the peerreviewed and grey literature. ${ }^{112143446364}$ A 2018 review by the National Academies of Sciences, Engineering, and Medicine on the public health consequences of e-cigarettes concludes that there is substantial evidence that e-cigarette use increases risk of ever using combustible tobacco cigarettes, and moderate evidence that e-cigarette use increases the frequency and intensity of subsequent combustible tobacco smoking, among youth and young adults. ${ }^{64}$ Previous systematic reviews have focused on evidence in those 30 years of age or less, whereas our review included data on adults and former smokers. This is the first systematic review to examine whether e-cigarette use is associated with smoking relapse. 
The use of e-cigarettes may represent a risk factor for cigarette smoking initiation, current smoking and relapse to cigarette smoking for several behavioural and physiological reasons. For those who use nicotinedelivering e-cigarettes, a resulting addiction to nicotine may leave users at risk of seeking other forms of inhalable nicotine, such as combustible cigarettes. ${ }^{65} 66$ Additionally, as e-cigarettes can mimic behavioural (eg, hand-mouth) and sensory (eg, taste) aspects of smoking, associated e-cigarette habits and movements may make the transition to combustible smoking more natural. ${ }^{6768}$ Further studies should examine potential mediators to better understand possible mechanisms for the association between e-cigarette use and subsequent cigarette use. Although one study showed that an intervention designed to reduce smoking initiation in adolescents through self-regulatory implementation intentions attenuated the odds of smoking uptake in never smokers who used e-cigarettes, a statistically significant increased odds remained. ${ }^{47}$

Although studies in this review were consistent in finding increased risks of smoking uptake in non-smokers exposed to e-cigarettes, the magnitude of this increased risk varied substantially between studies. The reason for this variation is unclear, but may relate to the different products, populations and policy environments. In addition, it is challenging to estimate the overall effect of e-cigarettes on smoking initiation due to the variety of ways in which devices (eg, e-cigarettes, JUULs, pods, vape pens) and users (eg, never-users, ever-users, current-users, former users) are classified. The high heterogeneity in most of the results from the metaanalyses suggests that pooled ORs should be interpreted as an average of disparate results, rather than a reflection of the true underlying effect.

A limitation in this review is that included studies were limited to those written in English. While emerging results from this review and similar studies provide evidence regarding the association between e-cigarette and combustible cigarette use, the evidence is heavily weighted towards US and UK data. Only nine countries were included in this analysis, with a notable lack of data from the Asia-Pacific, Africa and the Middle East. Furthermore, the studies were reliant on self-reported product use, which is likely to be subject to self-reporting bias. All three systematic reviews rated moderate in the AMSTAR 2 risk of bias assessment and the 12 newly identified studies rated between 5 and 8 on the NOS. Although the consistency of findings across multiple studies and settings supports the likelihood of a causal relationship, given the observational nature of many of the included studies, the findings may be potentially influenced by confounding factors, including socioeconomic status and the tendency for risk behaviours to occur together. As the ability to adjust for such confounding factors varied according to study, the possibility of residual confounding cannot be excluded.

\section{CONCLUSION}

This review found consistent evidence that use of e-cigarettes, largely nicotine-delivering, is associated with increased risk of subsequent combustible smoking initiation, current combustible smoking and smoking relapse after accounting for known demographic, psychosocial and behavioural risk factors. This is the first review to examine associations between e-cigarette use and cigarette use across the whole population, including youth, adults and former smokers. Intervention efforts and policies surrounding e-cigarettes are needed to reduce the potential of furthering combustible tobacco use in Australia and beyond.

Correction notice This article has been corrected since it was first published. The data in the abstract section has been modified.

Acknowledgements EB is supported by a Principal Research Fellowship from the National Health and Medical Research Council of Australia (reference: 1136128).

Contributors ONB, LF and EB all contributed to the study conception and design and interpretation of data. GJ and AY assisted with statistical analysis and interpretation of data. All authors were involved in revising the manuscript.

Funding This review was developed as part of an independent programme of work examining the health impacts of e-cigarettes, funded by the Australian Government Department of Health. EB is supported by the National Health and Medical Research Council of Australia (Principal Research Fellowship: 1136128).

Competing interests None declared.

Patient consent for publication Not required.

Provenance and peer review Not commissioned; externally peer reviewed.

Data availability statement All data used in the manuscript are from published research.

Supplemental material This content has been supplied by the author(s). It has not been vetted by BMJ Publishing Group Limited (BMJ) and may not have been peer-reviewed. Any opinions or recommendations discussed are solely those of the author(s) and are not endorsed by BMJ. BMJ disclaims all liability and responsibility arising from any reliance placed on the content. Where the content includes any translated material, BMJ does not warrant the accuracy and reliability of the translations (including but not limited to local regulations, clinical guidelines, terminology, drug names and drug dosages), and is not responsible for any error and/or omissions arising from translation and adaptation or otherwise.

Open access This is an open access article distributed in accordance with the Creative Commons Attribution Non Commercial (CC BY-NC 4.0) license, which permits others to distribute, remix, adapt, build upon this work non-commercially, and license their derivative works on different terms, provided the original work is properly cited, appropriate credit is given, any changes made indicated, and the use is non-commercial. See: http://creativecommons.org/licenses/by-nc/4.0/.

\section{ORCID iDs}

Olivia Nina Baenziger http://orcid.org/0000-0001-5100-7542

Amelia Yazidjoglou http://orcid.org/0000-0003-4406-368X

Grace Joshy http://orcid.org/0000-0002-0718-6368

Emily Banks http://orcid.org/0000-0002-4617-1302

\section{REFERENCES}

1 World Health Organization. Tobacco, 2019. Available: https://www. who.int/health-topics/tobacco [Accessed 31 Oct 2019].

2 Guerin NW, ASSAD V. Statistics \& trends: australian secondary students' use of tobacco, alcohol, over-the-counter drugs, and illicit substances. 2018. Melbourne: Cancer Council Victoria, 2017.

3 Australian Institute of Health and Welfare. Australian burden of disease study: impact and causes of illness and death in Australia 2015. Canberra: AlHW, 2019.

4 Farsalinos K. Electronic cigarettes: an aid in smoking cessation, or a new health hazard? Ther Adv Respir Dis 2018;12:1753465817744960. 
5 Chivers E, Janka M, Franklin P, et al. Nicotine and other potentially harmful compounds in "nicotine-free" e-cigarette liquids in Australia. Med J Aust 2019;210:127-8.

6 US Department of Health and Human Services. E-cigarette use among youth and young adults: a report of the Surgeon General. Atlanta, GA: Centres for Disease Control and Prevention, National Center for Chronic Disease Prevention and Health Promotion, Office on Smoking and Health, 2016.

7 Gorini G, Gallus S, Carreras G, et al. Prevalence of tobacco smoking and electronic cigarette use among adolescents in Italy: global youth tobacco surveys (GYTS), 2010, 2014, 2018. Prev Med 2020;131:105903.

8 Smith DM, Gawron M, Balwicki L, et al. Exclusive versus dual use of tobacco and electronic cigarettes among adolescents in Poland, 2010-2016. Addict Behav 2019;90:341-8.

9 Chen P-C, Chang L-C, Hsu C, et al. Dual use of e-cigarettes and traditional cigarettes among adolescents in Taiwan, 2014-2016. Nicotine Tob Res 2019;21:48-54.

10 Cho H-J, Dutra LM, Glantz SA. Differences in adolescent ecigarette and cigarette prevalence in two policy environments: South Korea and the United States. Nicotine Tobacco Research 2018;20:949-53.

11 McNeill A, Brose LS, Calder R, et al. Vaping in England: an evidence update including mental health and pregnancy, March 2020: a report commissioned by public health England. London: Public Health England, 2020.

12 AlHW. Australian Institute of health and welfare 2020. National drug strategy household survey 2019. Drug Statistics 2020.

13 Wamamili B, Wallace-Bell M, Richardson A, et al. Electronic cigarette use among university students aged 18-24 years in New Zealand: results of a 2018 national cross-sectional survey. BMJ Open 2020;10:e035093.

14 Patrick ME, Miech RA, Carlier C, et al. Self-reported reasons for vaping among 8th, 10th, and 12th graders in the US: Nationallyrepresentative results. Drug Alcohol Depend 2016;165:275-8.

15 Leavens ELS, Stevens EM, Brett El, et al. JUUL electronic cigarette use patterns, other tobacco product use, and reasons for use among ever users: results from a convenience sample. Addict Behav 2019;95:178-83.

16 Parker MA, Villanti AC. Patterns and frequency of current e-cigarette use in United States adults. Subst Use Misuse 2019;54:2075-81.

17 Dunbar MS, Davis JP, Rodriguez A, et al. Disentangling within- and between-person effects of shared risk factors on e-cigarette and cigarette use trajectories from late adolescence to young adulthood. Nicotine Tob Res 2019;21:1414-22.

$18 \mathrm{Kim} \mathrm{S}$, Selya AS. The relationship between electronic cigarette use and conventional cigarette smoking is largely attributable to shared risk factors. Nicotine Tob Res 2020;22:1123-30.

19 Glasser AM, Johnson AL, Niaura RS, et al. Youth vaping and tobacco use in context in the United States: results from the 2018 national youth tobacco survey. Nicotine \& Tobacco Research 2020.

20 Loukas A, Marti CN, Cooper M, et al. Exclusive e-cigarette use predicts cigarette initiation among college students. Addict Behav 2018;76:343-7.

21 Soneji S, Barrington-Trimis JL, Wills TA, et al. Association between initial use of e-cigarettes and subsequent cigarette smoking among adolescents and young adults: a systematic review and metaanalysis. JAMA Pediatr 2017;171:788-97.

22 Shea BJ, Reeves BC, Wells G, et al. AMSTAR 2: a critical appraisal tool for systematic reviews that include randomised or nonrandomised studies of healthcare interventions, or both. BMJ 2017;358:j4008.

23 Peterson J, Welch V, Losos M, et al. The Newcastle-Ottawa scale (NOS) for assessing the quality of nonrandomised studies in metaanalyses. Ottawa: Ottawa Hospital Research Institute, 2011.

24 Sterne JAC, Sutton AJ, loannidis JPA, et al. Recommendations for examining and interpreting funnel plot asymmetry in meta-analyses of randomised controlled trials. BMJ 2011;343:d4002.

25 Lee P, Fry J. Investigating gateway effects using the path study. F1000Res 2019;8:264

26 Berry KM, Fetterman JL, Benjamin EJ, et al. Association of electronic cigarette use with subsequent initiation of tobacco cigarettes in US youths. JAMA Netw Open 2019;2:e187794.

27 Chien Y-N, Gao W, Sanna M, et al. Electronic cigarette use and smoking initiation in Taiwan: evidence from the first prospective study in Asia. Int J Environ Res Public Health 2019;16 doi:10.3390/ ijerph16071145

28 Aleyan S, Gohari MR, Cole AG, et al. Exploring the bi-directional association between tobacco and e-cigarette use among youth in Canada. Int J Environ Res Public Health 2019;16 doi:10.3390/ ijerph16214256
29 Kinnunen JM, Ollila H, Minkkinen J, et al. Nicotine matters in predicting subsequent smoking after e-cigarette experimentation: a longitudinal study among Finnish adolescents. Drug Alcohol Depend 2019;201:182-7.

30 Brose LS, Bowen J, McNeill A, et al. Associations between vaping and relapse to smoking: preliminary findings from a longitudinal survey in the UK. Harm Reduct $J$ 2019;16:76.

31 Wills TA, Knight R, Sargent JD, et al. Longitudinal study of e-cigarette use and onset of cigarette smoking among high school students in Hawaii. Tob Control 2017;26:34-9.

32 Unger JB, Soto DW, Leventhal A. E-Cigarette use and subsequent cigarette and marijuana use among Hispanic young adults. Drug Alcohol Depend 2016;163:261-4.

33 Spindle TR, Hiler MM, Cooke ME, et al. Electronic cigarette use and uptake of cigarette smoking: a longitudinal examination of U.S. college students. Addict Behav 2017;67:66-72.

34 Primack BA, Soneji S, Stoolmiller M, et al. Progression to traditional cigarette smoking after electronic cigarette use among US adolescents and young adults. JAMA Pediatr 2015;169:1018-23.

35 Primack BA, Shensa A, Sidani JE, et al. Initiation of traditional cigarette smoking after electronic cigarette use among TobaccoNaïve US young adults. Am J Med 2018;131:443.e1-9.

36 Miech R, Patrick ME, O'Malley PM, et al. E-cigarette use as a predictor of cigarette smoking: results from a 1-year followup of a national sample of 12 th grade students. Tob Control 2017;26:e106-11.

37 Leventhal AM, Strong DR, Kirkpatrick MG, et al. Association of electronic cigarette use with initiation of Combustible tobacco product smoking in early adolescence. JAMA 2015;314:700-7.

38 Barrington-Trimis JL, Kong G, Leventhal AM, et al. E-cigarette use and subsequent smoking frequency among adolescents. Pediatrics 2018;142 doi:10.1542/peds.2018-0486

39 East K, Hitchman SC, Bakolis I, et al. The association between smoking and electronic cigarette use in a cohort of young people. J Adolescent Health 2018;62:539-47. doi:10.1016/j. jadohealth.2017.11.301

40 Best C, Haseen F, Currie D, et al. Relationship between trying an electronic cigarette and subsequent cigarette experimentation in Scottish adolescents: a cohort study. Tobacco Control 2018;27:373-8

41 Lozano P, Barrientos-Gutierrez I, Arillo-Santillan E, et al. A longitudinal study of electronic cigarette use and onset of conventional cigarette smoking and marijuana use among Mexican adolescents. Drug Alcohol Depend 2017:180:427-30.

42 Treur JL, Rozema AD, Mathijssen JJP, et al. E-cigarette and waterpipe use in two adolescent cohorts: cross-sectional and longitudinal associations with conventional cigarette smoking. Eur $J$ Epidemiol 2018;33:323-34.

43 Khouja JN, Suddell SF, Peters SE. Is e-cigarette use in nonsmoking young adults associated with later smoking? A systematic review and meta-analysis. Tob Control 2020:15. doi:10.1136/ tobaccocontrol-2019-055433

44 Aladeokin A, Haighton C. Is adolescent e-cigarette use associated with smoking in the United Kingdom?: a systematic review with meta-analysis. Tobacco Prevent Cessat 2019;5.

45 Bold KW, Kong G, Camenga DR, et al. Trajectories of e-cigarette and conventional cigarette use among youth. Pediatrics 2018;141 doi:10.1542/peds.2017-1832

46 Pénzes M, Foley KL, Nădășan V, et al. Bidirectional associations of e-cigarette, conventional cigarette and waterpipe experimentation among adolescents: a cross-lagged model. Addict Behav 2018;80:59-64.

47 Conner M, Grogan S, Simms-Ellis R, et al. Evidence that an intervention weakens the relationship between adolescent electronic cigarette use and tobacco smoking: a 24-month prospective study. Tob Control 2020;29:425-31.

48 Barrington-Trimis JL, Bello MS, Liu F, et al. Ethnic differences in patterns of cigarette and e-cigarette use over time among adolescents. J Adolesc Health 2019;65:359-65.

49 Osibogun O, Bursac Z, Maziak W. E-Cigarette use and regular cigarette smoking among youth: population assessment of tobacco and health study (2013-2016). Am J Prev Med 2020;58:657-65.

50 McMillen R, Klein JD, Wilson K, et al. E-Cigarette use and future cigarette initiation among never smokers and relapse among former smokers in the path study. Public Health Rep 2019;134:528-36.

51 Dai H, Leventhal AM. Association of electronic cigarette vaping and subsequent smoking relapse among former smokers. Drug Alcohol Depend 2019;199:10-17.

52 Watkins SL, Glantz SA, Chaffee BW. Association of Noncigarette tobacco product use with future cigarette smoking among youth in 
the population assessment of tobacco and health (path) study, 20132015. JAMA Pediatr 2018;172:181-7.

53 Hammond D, Reid JL, Cole AG, et al. Electronic cigarette use and smoking initiation among youth: a longitudinal cohort study. CMAJ 2017;189:E1328-36.

54 Conner M, Grogan S, Simms-Ellis R, et al. Do electronic cigarettes increase cigarette smoking in UK adolescents? Evidence from a 12-month prospective study. Tobacco Control 2018;27:365-72.

55 Auf R, Trepka MJ, Selim M, et al. E-Cigarette use is associated with other tobacco use among US adolescents. Int $J$ Public Health 2019;64:125-34.

56 Morgenstern M, Nies A, Goecke M, et al. E-cigarettes and the use of conventional cigarettes. Dtsch Arztebl Int 2018;115:243-8.

57 Moore GF, Littlecott HJ, Moore L, et al. E-cigarette use and intentions to smoke among 10-11-year-old never-smokers in Wales. Tob Control 2016;25:147-52.

58 Eastwood B, Dockrell MJ, Arnott D, et al. Electronic cigarette use in young people in Great Britain 2013-2014. Public Health 2015;129:1150-6.

59 de Lacy E, Fletcher A, Hewitt G, et al. Cross-sectional study examining the prevalence, correlates and sequencing of electronic cigarette and tobacco use among 11-16-year olds in schools in Wales. BMJ Open 2017;7:e012784.

60 Moore G, Hewitt G, Evans J, et al. Electronic-cigarette use among young people in Wales: evidence from two cross-sectional surveys. BMJ Open 2015;5:e007072.
61 Hughes K, Bellis MA, Hardcastle KA, et al. Associations between ecigarette access and smoking and drinking behaviours in teenagers. BMC Public Health 2015;15:244.

62 Barrington-Trimis JL, Urman R, Berhane K, et al. E-cigarettes and future cigarette use. Pediatrics 2016;138 doi:10.1542/peds.20160379

63 Byrne S, Brindal E, Williams G. E-cigarettes, smoking and health. A literature review update Australia. CSIRO, 2018.

64 National Academies of Sciences, Engineering, and Medicine. Public health consequences of e-cigarettes. Washington, DC: National Academies Press, 2018.

65 Owusu D, Huang J, Weaver SR, et al. Patterns and trends of dual use of e-cigarettes and cigarettes among U.S. adults, 2015-2018. Prev Med Rep 2019;16:101009.

66 Rapp JL, Alpert N, Flores RM, et al. Serum cotinine levels and nicotine addiction potential of e-cigarettes: an NHANES analysis. Carcinogenesis 2020;41:1454-9.

67 Dawkins L, Munafò M, Christoforou G, et al. The effects of ecigarette visual appearance on craving and withdrawal symptoms in abstinent smokers. Psychol Addict Behav 2016;30:101-5.

68 Zare S, Nemati M, Zheng Y. A systematic review of consumer preference for e-cigarette attributes: flavor, nicotine strength, and type. PLoS One 2018;13:e0194145. 